\title{
Terapia cognitivo-comportamental em grupo para a disfunção sexual na pós-menopausa
}

\author{
Group cognitive behavioral therapy for female \\ sexual dysfunction in postmenopause
}

Maria de Jesus Siqueira de Almeida', Clayton Peixoto², Tatiana Teixeira de Siqueira Bilememjian Ribeiro', Lucy Maria da Silva', André Barciela Veras ${ }^{1,2}$

\section{RESUMO}

Objetivo: Validação de protocolo de terapia cognitivo-comportamental breve em grupo para disfunção sexual feminina na pós-menopausa. Métodos: Intervenção em grupo com mulheres ( $n=14$ ) na pós-menopausa (55 a 75 anos) com disfunção sexual, em acompanhamento ginecológico ambulatorial em um centro especializado para queixas relacionadas ao climatério. As pacientes foram divididas em grupos: o primeiro (Grupo Teste, $\mathrm{n}=5$ ) recebeu abordagem cognitivo-comportamental de 12 sessões. Os dois grupos (denominados igualmente Grupo Intervenção $-n=5$ e $n=4$ ) foram formados pela divisão dos sujeitos para melhor acomodação física e receberam 10 sessões, resultantes de ajuste e mensuração dos procedimentos do protocolo a partir do Grupo Teste. Foram aplicados a Escala de Índice de Funcionamento Sexual Feminino e o Questionário de Crenças Sexuais Disfuncionais antes e depois da intervenção. Resultados: Houve frequente ocorrência de comportamentos/crenças de baixa autoestima, problemas com o parceiro e desinteresse ou dificuldade sexual. A disfunção sexual (problemas com o desejo e/ou lubrificação, orgasmo, satisfação e dor) e as crenças sexuais disfuncionais (conservadorismo, pecado, idade-crenças, imagem corporal, afetividade e maternidade) apresentaram melhora significativa com a aplicação do protoco-

\section{Palavras-chave}

Terapia cognitivo-comportamental, menopausa, disfunção sexual, crenças sexuais. lo. Conclusão: Considerando os aspectos particulares das mulheres com disfunção sexual na pós-menopausa, desenvolvemos um protocolo de terapia cognitivo-comportamental em grupo, com significativo potencial terapêutico. Embora não tenha sido utilizado um grupo controle e tenha sido aplicado em número reduzido de participantes, esse protocolo pode ser avaliado e utilizado em casos similares, cabendo novas pesquisas para verificar a aplicabilidade dele para disfunção sexual nesse estágio do desenvolvimento feminino.

\section{ABSTRACT}

Objective: Validation of the protocol of brief cognitive behavioural group therapy for female sexual dysfunction on post menopause. Methods: Group intervention with postmenopausal (55-75 years old) women $(n=14)$ with sexual dysfunction, on gynecological outpatient follow-up in a specialized center for climacteric related complaints. The patients were divided into groups: the first (Test Group, $\mathrm{n}=5$ ) received 12 sessions of cognitivebehavioral approach. The two groups (equally named Intervention Group $-n=5$ and $n=4$ )

1 Universidade Católica Dom Bosco (UCDB), Campo Grande, MS, Brasil. 2 Universidade Federal do Rio de Janeiro (UFRJ), Instituto de Psiquiatria, Rio de Janeiro, RJ, Brasil.

Endereço para correspondência: Clayton Peixoto E-mail: claytonpeixoto@yahoo.com.br 


\section{Keywords}

Behavioral-cognitive

therapy, menopause, sexual

dysfunction, sexual beliefs. were formed by the division of subjects for bettering physical accommodation and received 10 session, resulting from adjustment and measurement of the protocol procedures starting from the Test Group. The Female Sexual Function Index scale and the Sexual Dysfunctional Beliefs Questionnaire were applied before and after intervention. Results: There were frequent reports of behaviors/beliefs of low self-esteem, problems with partner and sexual disinterest or difficulty. Sexual dysfunction (problems with desire and/or lubrication, orgasm, satisfaction and pain) and dysfunctional sexual beliefs (conservatism, sin, age-beliefs, body image, affectivity and maternity) both presented significant improvement with the protocol application. Conclusion: Considering the particular aspects of postmenopausal women with sexual dysfunction, we developed a cognitive behavioral group therapy protocol, with significant therapeutic potential. Though a control group was not used and that the application was done in a reduced number of participants, this protocol can be assessed and utilized in similar cases, being fit new researches to verify the applicability of such for sexual dysfunction at this stage of female development.

\section{INTRODUÇÃO}

A sexualidade na vida madura envolve (re)descobertas e mudanças hormonais e psicológicas. O desconforto ou incompreensão sobre essas alterações orgânicas e não orgânicas levam mulheres ao abandono ou dificuldades com o sexo. Um terço da vida feminina ocorre na pós-menopausa e o sexo integra a qualidade de vida, valorizando a reabilitação sexual'.

A disfunção sexual feminina envolve transtornos do interesse sexual, excitação, orgasmo, dor genitopélvica com a penetração e as disfunções induzidas por substâncias ou medicamentos e as não classificadas². Reduz o interesse sexual e causa dificuldades na excitação subjetiva e/ou genital que afetam o relaxamento vaginal para a penetração3. É frequente na menopausa e após ela e ocasiona sentimentos de inferioridade, angústia, depressão, insegurança, vergonha do corpo, baixa autoconfiança e crenças disfuncionais sobre a capacidade de provocar desejo no parceiro ${ }^{4}$.

O tratamento da disfunção envolve os afetos femininos, pensamentos, comportamentos e informação sobre o corpo. Os tabus morais são trabalhados para o desenvolvimento da atração, estimulação adequada e função sexual, com maior compreensão e conhecimento sobre a relação íntima da mulher com seu corpo e parceiro ${ }^{5}$.

As crenças são "compreensões duradouras tão fundamentais e profundas que frequentemente não são articuladas nem para si mesma. A pessoa considera essas ideias como verdades absolutas" ${ }^{\prime \prime}$. Na disfunção sexual feminina, a terapia cognitivo-comportamental se focaliza na positividade das crenças individuais e intervém pela redução ou resolução dos fatores subjetivos ${ }^{5}$. Age para plena vivência sexual nas várias fases da vida, inclusive a pós-menopausa, fase de reconstrução da experiência sexual feminina após o fim da fertilidade ${ }^{1,3,6,7}$.

Pela importância de seu tratamento nesse período-marco feminino e ausência de um protocolo para uso em grandes populações, este estudo tem como objetivo desenvolver um protocolo-piloto de tratamento da disfunção sexual feminina na pós-menopausa com base na terapia cognitivo-comportamental. Trata-se de estudo clínico de intervenção para avaliação da eficácia da terapia com procedimentos sistematizados em uma amostra brasileira de mulheres usuárias de um ambulatório público de atendimento ginecológico e ao climatério.

\section{MÉTODOS}

O grupo de sujeitos original da pesquisa foi formado por mulheres ( $n=19)$ na faixa etária de 55 a 75 anos, na pós-menopausa, em tratamento no Ambulatório de Climatério do Centro de Atenção à Mulher do Centro de Especialidades Médicas de Campo Grande, MS, Brasil. As participantes foram indicadas pela ginecologista ambulatorial e passaram por entrevista de avaliação prévia para identificação da disfunção sexual feminina e assinatura de consentimento de integração (TCLE).

Durante a aplicação/avaliação/reaplicação do protocolo, cinco participantes não concluíram as sessões por razões familiares. O estudo relata os resultados referentes às mulheres que concluíram as sessões $(n=14)$, distribuídas em três grupos: Grupo Teste $(n=5)$ e Grupo de Intervenção $(n=9)$, separado em dois subgrupos para melhor acomodação física $(n=5$ e $n=4)$.

Os instrumentos foram ficha de identificação/dados sociodemográficos, Índice de Funcionamento Sexual Feminino e Questionário de Crenças Sexuais Disfuncionais - Versão Feminina, estes dois últimos coletados pré e pós-intervenção?.

O Índice de Funcionamento Sexual Feminino foi aplicado para conhecimento das funções sexuais em seis domínios: desejo (questões 1-2), excitação (questões 3-6), lubrificação (questões 7-10), orgasmo (perguntas 11-13), satisfação (questões 14-16) e dor (questões 17-19). A pontuação por domínio varia de 1 a 6 - se inferior a 3,9 em um item indica disfunção sexual. O Questionário de Crenças Sexuais Disfun- 
cionais serve à avaliação de estereótipos e crenças sexuais específicas associadas à disfunção sexual ${ }^{8-10}$.

A terapia cognitivo-comportamental foi aplicada da seguinte forma: Grupo Teste $(n=5)$ recebeu 12 sessões, uma por semana. Houve necessidade de reestruturação da duração pela dificuldade de assiduidade na forma original, resultando em 10 sessões, uma por semana, para o Grupo de Intervenção.

O protocolo para o Grupo Teste (Figura 1) foi realizado com cronograma de estratégias para abordagem de temáticas envolvidas nas disfunções sexuais, elaborado e aplicado por profissional com experiência e habilitação em terapia cognitivo-comportamental (Maria de Jesus Siqueira Almeida, pesquisadora principal). Os temas foram definidos por médico com experiência em disfunção sexual feminina (André Barciela Veras) e pela pesquisadora principal. Com o consentimento das participantes, as sessões foram gravadas em dispositivo de áudio digital, transcritas e organizadas como fonte dos qualitativos. As sessões foram aplicadas ao Grupo Teste entre março e julho de 2015 e aos Grupos de Intervenção entre julho e novembro de 2015.

Os dados quantitativos foram analisados pelo tipo de variável: contínuas (média e desvio-padrão) e categóricas (frequência absoluta e relativa). As diferenças entre os grupos foram analisadas pelo teste $t$ e a entre as médias nos tempos T0 (pré-intervenção) e T1 (pós-intervenção), pelo teste t pareado. A significância estatística foi considerada para valores de $p<0,05$.

\section{RESULTADO}

Os temas abordados com maior frequência pelas voluntárias foram divididos em categorias a partir dos diálogos observados com o Grupo Teste, analisados com base em fundamentos teóricos e metodológicos que regem a terapia cognitivo-comportamental (Figura 2).

O protocolo (Figura 1) foi testado no Grupo de Intervenção, que apresentava semelhança com o Grupo Teste nos dados sociodemográficos (Tabela 1).

Os escores observados no Índice de Funcionamento Sexual Feminino e no Questionário de Crenças Sexuais Disfuncionais antes e depois da intervenção em todos os grupos (Tabelas 2 a 5) mostraram significativa elevação na pontuação média total do Índice de Funcionamento Sexual Feminino do Grupo Teste, com melhora nos índices de função sexual. Os principais aumentos ocorreram nos índices de desejo, satisfação, orgasmo e lubrificação vaginal. O maior valor no domínio de dor indica redução dele com igual significância, melhoria também constatada $(p<0,1)$ no domínio excitação (Tabela 2).

O Questionário de Crenças Sexuais Disfuncionais evidenciou significativa redução das crenças disfuncionais to- tais e das relacionadas ao conservadorismo, pecado, idade e imagem corporal. As ligadas à afetividade $(p=0,229)$ e à maternidade $(p=0,325)$ diminuíram discretamente, sem significância estatística (Tabela 3).

A comparação dos escores (Tabelas 4 e 5) apresenta meIhor visualização do funcionamento sexual feminino e das crenças sexuais antes e depois da intervenção. Houve meIhora significativa da função sexual e qualidade nos diferentes domínios no Grupo de Intervenção, exceto o domínio da dor $(p=0,11)$ (Tabela 4).

O Grupo de Intervenção evidenciou redução das crenças disfuncionais totais e das relacionadas ao conservadorismo, pecado e imagem corporal. As ligadas à maternidade e à afetividade não apresentaram modificações significativas (Tabela 5).

\section{DISCUSSÃO}

Os dados qualitativos reportam queixas físicas e emocionais femininas comuns, em especial sofrimento para adaptar/ inserir o sexo na vida madura; angústia/medo; depressão e ansiedade sobre parceiros e relacionamentos; ressecamento vaginal; dor com a penetração; redução do interesse sexual e ardência com a penetração, entre outras, reforços confirmativos da disfunção sexual. Segundo Bastos ${ }^{11}$, são queixas que podem ser resultado ou reflexo do acúmulo de papéis sobre a mulher moderna (preocupações no trabalho, afazeres domésticos e gestão do papel de mãe/esposa presente), que causam cansaço e estresse e impactam a sexualidade.

A análise do Índice de Funcionamento Sexual Feminino e do Questionário de Crenças Sexuais Disfuncionais pré e pós-intervenção em ambos os grupos mostra significativa resposta pós-procedimento. Dattilio e Freeman ${ }^{12}$ relatam que a terapia cognitivo-comportamental implica empatia com o paciente para identificação, avaliação e levantamento dos esquemas cognitivos para identificação de recursos e plano de ação para novas cognições e comportamentos. Os dados do Índice de Funcionamento Sexual Feminino indicaram modificação significativa no tempo 1 (após) em relação ao tempo 0 (antes) da intervenção. O aumento do escore total em orgasmo, satisfação e dor foi consideravelmente elevado.

Pesquisa recente de Bober et al..$^{13}$ voltada a mulheres previamente submetidas à salpingooforectomia de redução de risco indica melhoria significativa da função sexual, com aumento do escore total do índice de função sexual feminina ( $p=0,018)$ e subescalas desejo $(p=0,003)$, excitação $(p=0,003)$, satisfação $(p=0,028)$ e dor $(p=0,018)$ após a terapia cognitivo-comportamental. As pontuações médias do Brief Symptom Inventory 18 (Inventário Breve de Sintomas) reduziram significativamente para a gravidade global $(p<0,001)$, escalas de somatização $(p=0,029)$ e ansiedade $(p<0,001)$, sinalizando melhora do sofrimento psíquico. 
Síntese das técnicas e conteúdos para 10 sessões do modelo de TCC para disfunção sexual na pós-menopausa*

Sessöes Conteúdo Técnica

$1^{\text {a }} \quad$ Apresentação e interação do grupo. Conhecer e fazer conhecer cada membro, aliança terapêutica.

Discussão sobre o conceito de menopausa.

Esclarecimento dos conceitos disfuncionais e informação adicional sobre a temática com texto de Dr. Dráuzio Varella ou outro à escolha que seja suficientemente completo e adequado ao grupo. Aplicação do Questionário do IFSF.

Dinâmica de Interação (coração partido). Rosset (2010).

Tarefa: Texto de Dr. Dráuzio Varella -

Menopausa ou similar.

Escala IFSF.

$2^{\text {a }} \quad$ Psicoeducação sobre a menopausa.

Estabelecimento de relação com a sessão anterior após ouvir sobre a tarefa de casa. Qual o entendimento de cada uma da leitura? Leitura: Eventos comuns da menopausa (Dattilio e Freeman, 1995).

Abordagem dos sintomas da menopausa.

3a Disfunções sexuais na menopausa.

Esclarecer conceitos e informar adicionalmente sobre os problemas sexuais da pós-menopausa. Explicar as doenças que podem incidir ou se manifestar com mais intensidade. Comentar o transtorno hipoativo, transtorno de aversão ao sexo, transtorno orgásmico feminino ou anorgasmia, dispareunia (dor genital associada à relação sexual), vaginismo (DSM-5) (APA, 2014).

Coletar informações adicionais sobre a dificuldade sexual individualmente. Tarefa de casa: leitura do texto sobre disfunções sexuais.

Apresentação do Modelo Cognitivo-Comportamental (TCC), com foco inicial no pensamento e, em seguida, no sentimento e no comportamento.

Pensamento distorcido comum a todos os distúrbios psicológicos.

Ensinar a fazer o P-S-C, registro de pensamentos para educar o paciente pela TCC a identificar, avaliar e modificar pensamentos

disfuncionais. Desmistificar as crenças, mitos, preconceitos e tabus relacionados à menopausa.

Tarefa de casa: conforme surgir no grupo a necessidade confrontar, treinar o RPD durante a semana.

5a Crenças negativas da imagem corporal; identificar emoçōes por meio de jogos com balōes (Rosset, 2010); identificar as necessidades afetivas de cada uma; respiração diafragmática (Ito et al., 1998).

Identificar a autoimagem negativa individual de cada participante e complexos de inferioridade. Tarefa de casa: escrever um texto com o título Como vocêse vê, sobre como imagina que os outros Ihe veem. Resumo final e feedback (devolutiva).

6a Relaxamento progressivo de Jacobson (Jacobson, 1964) e autoestima.

Feedback da tarefa de Como cada uma se vê, técnica de autorreconhecimento corporal (Rosset, 2010) e autoexame por meio da imagem no espelho.

7a Percepção das zonas erógenas do corpo.

Contato, estimulação sensorial.

Identificação do conhecimento sobre o outro; 0 que 0 companheiro sabe sobre as necessidades emocionais.

Trabalhar com o texto Uma história de carícias, de Claude Steiner, adaptado por Shinyashiki (1992).

Localizar as regiões mais excitantes e prazerosas do corpo, explorar o próprio corpo, sem incluir os genitais e se permitir conhecer as regiões erógenas e o prazer sensorial para 0 bem-estar e o relaxamento.

Resumo e feedback (devolutiva).

$8^{\mathrm{a}} \quad$ Conversa informal sobre a relação sexual e o parceiro (Caballo, 2007).

- Como é a relação com o parceiro, o que gosta e não gosta, contato e estimulação sensoriais;

- 0 que ele sabe sobre as necessidades emocionais;

- Uso dos quadrantes (gosto e faço, gosto e não faço, não gosto e faço e não gosto e não faço).

- Técnica do roley playing;

- Trabalhar as crenças sobre a masturbação que surgirem no grupo (Caballo, 2007).

Tarefa: regras e etiquetas para o diálogo (Beck, 1995).

9a

Masturbação

Trabalhar as crenças que o grupo apresentar sobre 0 tema (Caballo, 2007).

Explicar a importância da masturbação, da exploração manual dos genitais e concentração nas regiões mais excitantes e prazerosas, a fim do autoconhecimento e orgasmo. Explorar o próprio corpo sem incluir os genitais e reconhecer as regiões erógenas e prazer sensorial para 0 bem-estar e relaxamento. Identificar 0 que pensam sobre masturbação, desmistificar as crenças e orientar nas formas de autoaprendizado para 0 orgasmo.

10 $\quad$ Encerramento com avaliação e devolutiva

Reaplicação da escala.

Estimular os pacientes a reconsiderarem o tempo que haviam reservado à sessão de terapia, como tempo para continuar cuidando de sua sexualidade depois do fim da intervenção (Caballo, 2007).

Psicoeducação por meio de slides. Escuta de cada participante e de seus sintomas.

Biblioterapia, artigos.

Vídeos

Dinâmica

Conversa em pares

Aplicar o RPD

Discussão do grupo

Treinamento RPD

Socialização do grupo e educação sobre o modelo cognitivo P-S-C. Ficha RPD

Reestruturação cognitiva

Colocando a crença encontrada no balão no RPD

Técnica de relaxamento

Escuta, intervenções

Técnica do espelho

Caixa

Texto: Uma história de carícias

Técnica do carinho quente

Curtigrama - treino da comunicação Roley playing

Reestruturação cognitiva por meio da seta descendente

Autoexame genital

Roda de conversa para avaliação do programa

Aplicação da escala.

De IFSF.

Figura 1. Protocolo Final aplicado aos Grupos de Intervenção $(n=9)$ após a reformulação pós-intervenção com Grupo Teste $(n=5)$ (Campo Grande/MS - 2015). * Os autores utilizados para a elaboração do protocolo e nele mencionados são: American Psychiatric Association. APA. DMS-IV-TR - Manual Diagnóstico e Estatístico de Transtornos Mentais. 4a ed. Porto Alegre: Artes Médicas; 2002. Beck AT. Terapia cognitiva: teoria e prática. São Paulo: Artmed; 1995. Caballo VE. Manual de psicologia clínica infantil e do adolescente. São Paulo: Editora Santos; 2007. Dattilio FM, Freeman A. Estratégias cognitivo-comportamentais para intervenção em crises: tópicos especiais. Campinas: Psy; 1995. Ito LM, Lotufo Neto F, Roso CM, Wielenska R. Depressão. In: Ito LM. Terapia cognitivo-comportamental para transtornos psiquiátricos. São Paulo: Artes Médicas; 1998. Jacobson E. Progressive relaxation: a psychological and clinical investigation of muscular states and their significance in psychology and medical practice. Chicago: University of Chicago Press; 1938. Rosset SM. Técnica de psicoterapia relacional sistêmica. $4^{a}$ ed. Curitiba: Sol; 2010. Shinyashiki R. A carícia essencial: uma estória de carinhos. São Paulo: Gente; 1992. Fonte: O autor (2016). 


\begin{tabular}{lll}
\hline & \multicolumn{1}{c}{ Categorias } \\
\hline Categoria I & Vivência da sexualidade na menopausa & $\begin{array}{l}\text { Modo como cada mulher vivencia o sexo e qual a natureza de suas experiências (se positivas ou negativas). } \\
\text { Procura conhecer o estado de sua libido, a qualidade emocional das relações e como as mulheres relacionam a sua } \\
\text { sexualidade ao climatério. }\end{array}$ \\
Categoria II & $\begin{array}{l}\text { Visão sobre si mesma no período da } \\
\text { menopausa }\end{array}$ & $\begin{array}{l}\text { Avaliação da autoimagem da mulher e sua leitura perante seus conceitos de sexualidade, feminilidade, autoestima } \\
\text { e no complexo processo de envelhecimento. }\end{array}$ \\
Categoria III & Preocupação e dificuldades com o parceiro & $\begin{array}{l}\text { Visões e percepções das mulheres acerca da relação com o parceiro: a qualidade, a saúde, o relacionamento, a } \\
\text { comunicação e a visão do outro, inclusive no que tange à atração e envolvimento físico, sexual, emocional e afetivo. }\end{array}$ \\
Categoria IV & $\begin{array}{l}\text { Sentimentos da mulher com DSF } \\
\text { preferencialmente na pós-menopausa }\end{array}$ & $\begin{array}{l}\text { Visões e sentimentos da mulher que tem essa experiência e como ela observa sua evolução durante a TCC. Os } \\
\text { principais interesses são aspectos como autoestima, autonomia e autocuidado da satisfação sexual. }\end{array}$ \\
\hline
\end{tabular}

Figura 2. Protocolo Final aplicado no Grupo de Intervenção $(n=9)$ após a reformulação decorrente das observações iniciais no Grupo Teste $(n=5)$. Fonte: $O$ autor (2016).

Tabela 1. Dados sociodemográficos do Grupo Teste e Grupo Intervenção

\begin{tabular}{|c|c|c|c|}
\hline Dados & Grupo Teste & Grupo Intervenção & Pvalor \\
\hline Idade & 59,0 & 55,5 & NS \\
\hline \multicolumn{4}{|l|}{ Religião } \\
\hline Católica & $40,0 \%$ & $33,3 \%$ & \multirow[t]{3}{*}{ NS } \\
\hline Evangélica & $40,0 \%$ & $55,5 \%$ & \\
\hline Nenhuma & $20,0 \%$ & $11,1 \%$ & \\
\hline \multicolumn{4}{|l|}{ Profissão } \\
\hline Aposentada & $20,0 \%$ & $22,2 \%$ & \multirow[t]{3}{*}{ NS } \\
\hline Do lar & $20,0 \%$ & $33,0 \%$ & \\
\hline Liberal & $60,0 \%$ & $44,0 \%$ & \\
\hline \multicolumn{4}{|l|}{ Estado civil } \\
\hline Separada & $20,0 \%$ & $22,2 \%$ & \multirow[t]{4}{*}{ NS } \\
\hline Casada & $80,0 \%$ & $44,4 \%$ & \\
\hline Viúva & 0,0 & $11,1 \%$ & \\
\hline Divorciada & 0,0 & $22,2 \%$ & \\
\hline
\end{tabular}

* Grupo Teste. ${ }^{* *}$ Grupo intervenção. NS: diferença não estatisticamente significativa.

Fonte: 0 autor (2016).

Tabela 2. Comparação dos escores do Índice de Funcionamento Sexual Feminino do Grupo Teste $(n=5)$

\begin{tabular}{lccccc}
\hline Domínios & $\begin{array}{c}\text { Tempo } \\
\text { Média (DP) }\end{array}$ & $\begin{array}{c}\text { Tempo } 1 \\
\text { Média (DP) }\end{array}$ & Diferença média & Intervalo de confiança & Valor deP \\
\hline Desejo & $2,04(0,53)$ & $3,84(0,32)$ & $-1,80(0,42)$ & $-2,32 /-1,2$ & $-2,33 / 0,1$ \\
Excitação & $2,22(1,82)$ & $3,30(0,92)$ & $-1,08(1,00)$ & $2,77 /-0,3$ & 0,001 \\
Lubrificação & $2,04(0,57)$ & $3,60(1,21)$ & $-1,56(0,98)$ & $-3,05 / 0,9$ & 0,024 \\
Orgasmo & $1,84(0,66)$ & $3,84(1,28)$ & $-2,00(0,84)$ & $-2,99 / 1,4$ & 0,006 \\
Satisfação & $1,44(0,72)$ & $3,68(1,21)$ & $-2,24(0,60)$ & $-2,96 / 0,2$ & 0,001 \\
Dor & $1,76(1,34)$ & $3,36(1,91)$ & $-1,600(1,09)$ & $-14,1 /-6,3$ & 0,031 \\
Total & $11,34(4,48)$ & $21,62(6,06)$ & $-10,28(3,14)$ & & 0,002 \\
\hline
\end{tabular}

* DP: desvio-padrão.

Fonte: 0 autor (2016). 
A autoeficácia feminina no controle dos efeitos colaterais da salpingooforectomia de redução de risco aferida pela escala de autoeficácia sexual foi significante $(p<0,001)$. O conhecimento dos efeitos colaterais sexuais melhorou comparado ao início da intervenção $(p<0,001)$.

Lerner ${ }^{14}$ observou, entre mulheres com queixa de disfunção sexual submetidas à terapia cognitivo-comportamental ( $n=106$, idade média de 39,56 anos $- \pm 10,15$ anos), atendidas no Ambulatório de Medicina Sexual do Hospital das Clínicas da Faculdade de Medicina da Universidade de São Paulo, entre os anos de 2006 a 2009, Quociente de Satisfação Sexual Feminina total médio de 18,08 e melhora significati- va na função sexual em comparação a grupo controle que não recebeu a abordagem. Os resultados sinalizam para o benefício da terapia na disfunção sexual se comparada ao tratamento expectante, em especial pela mudança sobre pensamentos disfuncionais associados à prática sexual, importantes pela necessidade que o grupo apresenta de elaboração das percepções de fracasso, negação, insatisfação e falta de pensamentos eróticos. Os resultados demostraram eficácia da terapia cognitivo-comportamental no grupo estudado. Devido ao aumento de pacientes constatado pelo pesquisador, foi sugerido seu uso em ambientes de saúde pública.

Tabela 3. Comparação da escala do Questionário de Crenças Sexuais Disfuncionais do Grupo Teste $(n=5)$

\begin{tabular}{|c|c|c|c|c|c|}
\hline Domínios & $\begin{array}{c}\text { Tempoo } \\
\text { Média (DP) }\end{array}$ & $\begin{array}{c}\text { Tempo } 1 \\
\text { Média (DP) }\end{array}$ & Diferença média & Intervalo de confiança & Valor de $P$ \\
\hline Conservadorismo & $33,80(3,11)$ & $21,80(3,11)$ & $12,00(2,12)$ & $9,36 / 14,63$ & $<0,001$ \\
\hline Pecado & $16,20(2,77)$ & $9,20(4,65)$ & $7,00(5,70)$ & $-0,078 / 14,07$ & 0,052 \\
\hline Idade - crenças & $17,40(1,67)$ & $9,40(3,05)$ & $5,60(2,07)$ & $3,02 / 8,17$ & 0,004 \\
\hline Imagem corporal & $11,80(2,28)$ & $6,20(2,28)$ & $5,60(2,07)$ & $3,02 / 8,17$ & 0,004 \\
\hline Afetividade & $20,40(2,19)$ & $22,80(3,27)$ & $-2,40(3,78)$ & $-7,09 / 2,29$ & 0,229 \\
\hline Maternidade & $11,60(1,94)$ & $10,20(1,64)$ & $1,40(2,79)$ & $-2,06 / 4,86$ & 0,325 \\
\hline Total & $90,80(6,38)$ & $56,80(10,60)$ & $34,00(13,43)$ & $17,31 / 50,68$ & 0,005 \\
\hline
\end{tabular}

Fonte: 0 autor (2016).

Tabela 4. Comparação dos escores dos testes de funcionamento sexual e crenças femininas do Grupo de Intervenção $(n=9)$

\begin{tabular}{|c|c|c|c|c|c|}
\hline Domínios IFSF & $\begin{array}{c}\text { Tempo } 0 \\
\text { Média (DP) }\end{array}$ & $\begin{array}{c}\text { Tempo } 1 \\
\text { Média (DP) }\end{array}$ & Diferença média & Intervalo de confiança & Valor de $\mathrm{P}$ \\
\hline Desejo & $2,20(0,99)$ & $3,66(1,05)$ & $-1,46(0,90)$ & $-2,16 /-0,77$ & 0,001 \\
\hline Excitação & $1,20(1,11)$ & $3,03(1,48)$ & $-1,83(1,55)$ & $-3,02 /-0,63$ & 0,008 \\
\hline Lubrificação & $1,33(1,33)$ & $3,10(1,63)$ & $-1,76(1,80)$ & $-3,15 /-0,38$ & 0,019 \\
\hline Orgasmo & $1,51(1,42)$ & $3,82(1,65)$ & $-2,31(1,87)$ & $-3,75 /-0,87$ & 0,006 \\
\hline Satisfação & $1,73(1,41)$ & $3,15(1,46)$ & $-1,42(1,00)$ & $-2,19 /-0,65$ & 0,003 \\
\hline Dor & $1,46(2,09)$ & $1,77(2,29)$ & $-0,31(0,52)$ & $-0,71 / 0,08$ & 0,111 \\
\hline Total & $9,44(7,69)$ & $18,55(7,68)$ & $-9,11(6,24)$ & $-13,9 /-4,31$ & 0,002 \\
\hline
\end{tabular}

Fonte: 0 autor (2016).

Tabela 5. Comparação da escala do Questionário de Crenças Sexuais Disfuncionais do Grupo Intervenção $(n=9)$ - Campo Grande, MS, 2015

\begin{tabular}{lccccc}
\hline Domínios & $\begin{array}{c}\text { Tempo } \\
\text { Média (DP) }\end{array}$ & $\begin{array}{c}\text { Tempo } 1 \\
\text { Média (DP) }\end{array}$ & Diferença média & Intervalo de confiança & Valor de P \\
\hline Conservadorismo & $29,66(06,02)$ & $19,77(06.92)$ & $09,88(08,59)$ & $3,28 / 16,49$ & 0,009 \\
Pecado & $15,00(03,67)$ & $10,55(02,24)$ & $04,44(04,66)$ & $0,85 / 8,03$ & 0,021 \\
Idade - crenças & $17,77(05,04)$ & $11,77(03,45)$ & $06,00(07,31)$ & $0,37 / 11,62$ & 0,039 \\
Imagem corporal & $10,44(03,94)$ & $05,77(01,39)$ & $04,66(04,58)$ & $02,14 / 8,18$ & 0,016 \\
Afetividade & $23,00(02,95)$ & $20,33(02,87)$ & $01,66(04,71)$ & $-0,95 / 6,29$ & 0,128 \\
Maternidade & $11,55(02,85)$ & $10,33(01,80)$ & $01,22(03,36)$ & $-1,35 / 3,79$ & 0,305 \\
Total & $84,44(18,98)$ & $58,22(13,44)$ & $26,22(25,95)$ & $6,27 / 46,17$ & 0,016 \\
\hline
\end{tabular}

Fonte: 0 autor (2016). 
As características deste estudo quanto as pesquisas de Bober et al..$^{13}$ e Lerner ${ }^{14}$ se relacionam aos resultados satisfatórios da aplicação do protocolo elaborado. O Grupo Teste, que recebeu as intervenções integrais, teve elevação positiva em todos os quesitos básicos da satisfação e desempenho sexual. As elevações médias tiveram escores mínimos de 1,5 e máximos de 2,24, com valores de p confirmatórios da significância. Os maiores acréscimos ocorreram em orgasmo/desejo/lubrificação/satisfação.

A análise do Questionário de Crenças Sexuais Disfuncionais evidenciou redução significativa das crenças disfuncionais totais nos Grupos Teste e Intervenção, em especial nas de conservadorismo, pecado, idade e imagem corporal $(p<0,05)$. Não foram observadas melhoras significativas nas crenças sobre maternidade e afetividade, mas deve ser considerada a brevidade da intervenção e possíveis benefícios com maior período de tratamento.

O presente estudo repetiu o padrão de melhora sintomática descrito na literatura, a exemplo do estudo de Ter Kuile et al. ${ }^{15}$, em que pacientes com vaginismo $(n=70)$ foram divididas em dois grupos ( $n=35$ cada), e um deles $(n=45)$ recebeu terapia cognitivo-comportamental por três meses. Após 12 semanas, houve melhora na frequência das relações sexuais, nos sintomas de vaginismo e na dor durante o sexo, decorrentes da dissolução das crenças negativas relacionadas à penetração e à dor. Mas, diferentemente dessa pesquisa, nosso estudo não identificou redução na repulsa sexual. Essa diferença pode ter natureza cultural ou ser situação específica do grupo analisado, em que as mulheres se mostraram mais receptivas ao sexo, compreensivas, afetuosas e abertas ao diálogo com seus parceiros.

O estudo apresentou limitações, entre as quais um número restrito de participantes e a aplicação em um único conjunto avaliativo, sem utilização de controles. A limitação se deu pela condição de participação livre e espontânea no estudo, quando a indicação de mulheres pelo espaço de atendimento foi mais numerosa, porém o interesse em participar foi evidenciado apenas pela amostra composta no estudo.

Os resultados da aplicação do nosso protocolo permitem apontar na terapia cognitivo-comportamental em grupo uma série de recursos consideráveis que apoiam o empoderamento sexual feminino e têm validade para a resolução das disfunções sexuais, como apoio na transição para uma nova fase que se mostra cada vez mais extensa: a pós-menopausa.

\section{CONCLUSÕES}

O presente estudo reforçou o potencial da terapia cognitivo-comportamental na redução dos pensamentos disfuncionais e disfunção sexual em mulheres na pós-menopausa. 0 modelo de protocolo apresentado, breve e grupal, fortalece a viabilidade da terapia nos espaços de saúde pública. Tais fatores tornam promissor o teste controlado do protocolo elaborado neste estudo para o atendimento a grandes populações. Apesar da não utilização de um grupo controle, a base de resultados e de estudos teóricos reforça a terapia utilizada como método terapêutico funcional e de indicação sistemática para a disfunção sexual e outros problemas da sexualidade feminina. A relevância deste estudo se reforça na aplicação em grupo, um dos diferenciais para a inserção na saúde pública, que permite a construção conjunta de conhecimento, troca de experiências e confiança entre participantes e terapeuta. A comunicação possibilitou que, partindo dos relatos do grupo, as temáticas propostas fossem mais leves. Outros aspectos que contribuíram para o êxito da intervenção foram a execução no espaço físico de atendimento especializado e a contribuição da ginecologista na indicação do grupo para as pacientes.

\section{CONTRIBUIÇÕES INDIVIDUAIS}

Todos os autores atestam a contribuição conjunta na autoria, com atuação no desenvolvimento e pesquisa do material apresentado, conjuntamente e de modo paritário.

\section{CONFLITOS DE INTERESSE}

Os autores não possuem conflitos de interesse a serem declarados.

\section{AGRADECIMENTOS}

Agradecemos ao Centro de Especialidades Médicas/Centro de Atendimento a Mulher (CEM/CEAM), pelo apoio e auxílio durante o levantamento da pesquisa. Agradecemos a ao financiamento recebido pelo Conselho Nacional de Desenvolvimento Científico e Tecnológico (CNPq), pelo financiamento recebido, pelo sob o número 449309/2014-2.

\section{REFERÊNCIAS}

1. Blumel JE, Castelo Branco C, Chedraui PA, Binfa L, Dowlani B, Gomes MS, et al. Patients' and clinicians' attitudes after the Women's Health Initiative study. Menopause. 2004;11(1):57-61.

2. Martins RAS. Envelhecimento, atividade física e saúde cardiovascular. Coimbra: Faculdade de Ciências do Desperto e Educcã̧ão Física da Universidade de Coimbra; 2012.

3. Basson R, Berman J, Burnett A, Derogatis L, Ferguson D, Foucroy J, et al. Report of the international consensus development conference on female sexual dysfunction: definitions and classifications. J Urol. 2000;163(3):888-93.

4. Apollinário JC, Coutinho W, Póvoa LC, Meirelles R. Terapia hormonal e os sintomas psíquicos na menopausa. J Bras Psiquiatr. 1995;44(4):169-76.

5. Rangé B. Psicoterapias cognitivo-comportamentais: um diálogo coma psiquiatria. São Paulo: Artmed; 2001.

6. Beck AT, Rush AJ, Shaw BF, Emery G. Terapia cognitiva da depressão. Porto Alegre: Artmed; 1997; p. 52. 
7. Gouveia VV. A natureza motivacional dos valores humanos: evidências acerca de uma nova tipologia. Estud Psicol. 2003;8(3):431-43.

8. Heiman J, Lopiccolo J. Becoming orgasmic: a sexual personal growth program for women. New York: Prentice Hall; 1988.

9. Nobre PJ. Determinants of sexual desire problems in women: testing a cognitive emotional model. J Sex Marital Ther. 2009:35(5):1-18.

10. Nobre P, Gouveia JP, Gomes FA. Sexual dysfunctional beliefs questionnaire: an instrument to assess sexual dysfunctional beliefs as vulnerability factors to sexual problems. Sex Relationship Ther. 2003;18(2):101-35.

11. Bastos MH. Sorria, você está na menopausa: um manual de terapia natural para a mulher. São Paulo: Ground; 2001.
12. Dattilio FM, Freeman A. Estratégias cognitivo-comportamentais para intervenção em crises: tópicos especiais. Campinas: Editorial Psy; 1995.

13. Bober SL, Recklitis CJ, Bakan J, Garber JE, Patenaude AF. Addressing sexual dysfunction after risk-reducing salpingo-0ophorectomy: effects of a brief, psychosexual intervention. J Sex Med. 2015;12(1):189-97.

14. Lerner T. Terapia cognitivo-comportamental em grupo no tratamento de disfunções sexuais femininas [dissertação]. São Paulo: Universidade de São Paulo (USP); 2012.

15. Ter Kuile MM, Melles RJ, Tuijnman-Raasveld CC, de Groot HE, van Lankveld JJ. Therapist-Aided Exposure for Women with lifelong vaginismus: mediators of treatment outcome: a randomized waiting list control trial. J Sex Med. 2015;12(8):1807-19. 